IOS Press

\title{
Dynamic changes in practical inflammation and immunity markers in cancer patients receiving immune-enhancing nutritional supplementation during concurrent chemoradiotherapy
}

\author{
Nontiya Homkham ${ }^{\mathrm{a}}$, Pooriwat Muangwong ${ }^{\mathrm{b}, \mathrm{c}}$, Veeradej Pisprasert ${ }^{\mathrm{d}}$, Patrinee Traisathit ${ }^{\mathrm{e}}, \mathrm{f}$, \\ Rungarun Jiratrachu ${ }^{\mathrm{g}}$, Pattawee Chottaweesak ${ }^{\mathrm{h}}$ and Imjai Chitapanarux ${ }^{\mathrm{b}, \mathrm{c}, \mathrm{i}, *}$ \\ ${ }^{a}$ Faculty of Public Health, Thammasat University, Prathumthani, Thailand \\ ${ }^{\mathrm{b}}$ Division of Radiation Oncology, Faculty of Medicine, Chiang Mai University, Chiang Mai, Thailand \\ ${ }^{\mathrm{c}}$ Northern Thai Research Group of Radiation Oncology (NTRG-RO), Faculty of Medicine, Chiang Mai University, \\ Chiang Mai, Thailand \\ ${ }^{\mathrm{d}}$ Division of Clinical Nutrition, Faculty of Medicine, Khon Kaen University, Khon Kaen, Thailand \\ ${ }^{\mathrm{e}}$ Department of Statistics, Faculty of Science, Chiang Mai University, Chiang Mai, Thailand \\ ${ }^{\mathrm{f}}$ Center of Excellence in Bioresources for Agriculture, Industry and Medicine, Faculty of Science, Chiang Mai \\ University, Chiang Mai, Thailand \\ ${ }^{\mathrm{g}}$ Division of Radiation Oncology, Faculty of Medicine, Prince of Songkla University, Songkla, Thailand \\ ${ }^{\mathrm{h}}$ Radiotherapy Unit, Radiology Department, Maharat Nakhonratchasima Hospital, Nakhonratchasima, Thailand \\ ${ }^{\mathrm{i}}$ Chiang Mai Cancer Registry, Maharaj Nakorn Chiang Mai Hospital, Chiang Mai University, Chiang Mai, Thailand
}

Received 11 February 2021

Accepted 24 May 2021

\begin{abstract}
.
BACKGROUND: Immune-enhancing nutrition (IMN) strengthens the systematic inflammatory response and the immune system. Neutrophil to lymphocyte ratio (NLR) and absolute lymphocyte count (ALC) are affected during cancer therapies.

OBJECTIVE: We carried out an analysis of the dynamic changes in NLR and ALC over time in cancer patients with or without IMN supplementation.

METHODS: 88 cancer patients receiving concurrent chemoradiotherapy (CCRT) were randomized into regular diet group, and regular diet and IMN group.Generalized estimation equation models were used to assess associations between patient's characteristics, IMN, and dynamic changes in NLR and ALC over time.

RESULTS: NLR and ALC at pre-CCRT were significantly associated with dynamic changes in NLR (adjusted $\beta=1.08,95 \%$ confidence interval [CI]: 0.64-1.52) and ALC (adjusted $\beta=0.41,95 \%$ CI: 0.36-0.46). The magnitudes of the NLR and ALC changes through CCRT were lower in patients receiving IMN, although the differences were not statistically significant except ALC at the end of CCRT in head and neck cancer patients $(P=0.023)$.

CONCLUSION: Dynamic negative changes in both markers were demonstrated throughout CCRT. There were non-significant trend in promising changes in both NLR and ALC values in the whole group in IMN supplementation.
\end{abstract}

Keywords: Concurrent chemoradiotherapy, immune-enhancing nutrition, neutrophil to lymphocyte ratio, absolute lymphocyte count, cancer

\footnotetext{
* Corresponding author: Imjai Chitapanarux, Division of Radiation Oncology, Department of Radiology, Faculty of Medicine, Chiang
}

Mai University, 110 Intawarorose Road, Chiang Mai 50200, Thailand. Tel.: +66 5394 5456; E-mail: imjai.chitapanarux@cmu.ac.th. 


\section{Introduction}

The majority of patients receiving concurrent chemoradiotherapy (CCRT) have poor nutritional statuses [1$3]$ associated with deteriorated lymphoid tissue and subsequent weakening of the immune function [4]. In addition to the disease factor, CCRT itself also induces inflammation and immunological suppression that may cause other treatment-related toxicities [1-3].

The sufficiency of energy and protein intake is an important nutritional management option for patients receiving CCRT $[2,3]$. Nutritional supplementation improves several nutritional markers, such as albumin and prealbumin levels, lymphocyte count, etc. [5].

Immune-enhancing nutrition contains specific supplements that can moderate the inflammatory and immune responses $[3,6,7]$. Immune-enhancing nutritional supplementation is thought to strengthen the systematic inflammatory response and the immune system, which are known to be involved in the treatment response, progression, and prognosis in cancer patients [2,3,8,9]. A study on immune-enhancing nutrition has shown that dietary arginine, glutamine, and omega-3 fatty acid supplementation in immunocompromised patients (including cancer patients) improved immunity and decreased inflammation markers [10].

Although the inflammation response and immunity can be measured via many laboratory tests, the most sophisticated ones are not widely available in real-life practice. Complete blood count (CBC), a routine laboratory test accessible to most hospitals, can be used to evaluate inflammation and immunity [11]. The neutrophil to lymphocyte ratio (NLR; the percentage of neutrophils divided by the percentage of lymphocytes from a $\mathrm{CBC}$ test) is an inflammation-based score that is used as a predictor for the prognosis and survival of cancer patients. An increase in NLR is associated with poor prognosis and lower survival of head and neck, esophageal, and cervical cancers [12-16], and unfavorable outcomes in various other cancers [17]. In addition, NLR is associated with the nutritional status: a higher NLR is found in patients who are malnourished compared with those with a normal nutritional status [18].

Lymphocytes have an essential function in the cancer-related immune response, and the absolute lymphocyte count (ALC; the number of lymphocytes circulating in peripheral blood) is a prognostic factor for clinical outcomes of cancer patients [19-21]. Lymphopenia, a condition in which lymphocytes decrease to less than the normal value, is considered as a sign of im- munosuppression [22] and is an independent predictor of chemotherapy-associated toxicity as well as mortality [23-28]. Both radiotherapy and chemotherapy can negatively impact lymphocyte count [29-31]. In addition, patients with low ALC have a worse response to therapy and shorter progression-free survival [32-35].

In our previous study, nutrition counseling combined with immune-enhancing nutritional supplementation agents (arginine, glutamine, and fish oil) reduced the deterioration of nutritional status and also the severity of CCRT toxicity [8]. However, the relationship between immune-enhancing nutritional supplementation and practical inflammation and immunity markers NLR and ALC has rarely been studied in cancer patients undergoing CCRT. In this study, we carried out an analysis of the dynamic changes in NLR and ALC over time to evaluate the impact of immune-enhancing nutritional supplementation agents on cancer patients undergoing CCRT.

\section{Materials and methods}

\subsection{Patients}

We obtained data from our prospective analysis of patients enrolled in a multicenter, randomized control study conducting between December 2013 and February 2015 among three cancer centers: Chiang Mai University Hospital, Prince Songklanagarind University Hospital, and Maharat Nakhonratchasima Hospital [9]. Eighty-eight cancer patients who received definitive CCRT, including 40 head and neck cancer patients, 28 esophageal cancer patients, and 20 cervical cancer patients were included in the study. Head and neck cancer patients received 60-70 Gy concurrent with weekly cisplatin $40 \mathrm{mg} / \mathrm{m}^{2}$ or weekly carboplatin AUC 2, $50.4 \mathrm{~Gy}$. Esophageal cancer patients received 50.4 Gy concurrent with $75 \mathrm{mg} / \mathrm{m}^{2}$ of cisplatin on day 1 and $1,000 \mathrm{mg} / \mathrm{m}^{2}$ of fluorouracil on day $1-4$ every 28 days. Cervical cancer patients received 56 Gy concurrent with weekly $40 \mathrm{mg} / \mathrm{m}^{2}$ of cisplatin or weekly carboplatin AUC 2 plus brachytherapy 28 Gy in 4 fractions. Patients were randomly assigned to two groups: group A (control arm) receiving a regular diet with energy $1,500 \mathrm{kcal}$ and $60 \mathrm{~g}$ of protein per day, except esophageal cancer patients receiving blenderized nutrition with $2000 \mathrm{kcal}$ and $75 \mathrm{~g}$ of protein per day via feeding tube, and group $\mathrm{B}$ (study arm) receiving a regular diet with additional $500 \mathrm{kcal}$ per day of immune-enhancing nutritional supplementation containing arginine $6.16 \mathrm{~g}$, L-glutamine $3.07 \mathrm{~g}$, and fish oil $2.73 \mathrm{~g}$ that prepared in sachet form and provided 2 times per day during CCRT. 
Table 1

Median and interquartile range of the neutrophil to lymphocyte ratio and absolute lymphocyte count before and during CCRT

\begin{tabular}{|c|c|c|c|c|c|c|c|}
\hline & \multirow[b]{2}{*}{$n$} & \multicolumn{3}{|c|}{ NLR } & \multicolumn{3}{|c|}{ ALC } \\
\hline & & Median & IQR & $P$ & Median & IQR & $P$ \\
\hline All cancer sites $[n=88]$ & & & & $<0.001$ & & & $<0.001$ \\
\hline Pre-CCRT & 88 & 2.26 & 1.62 to 3.54 & & 2,070 & 1,723 to 2,420 & \\
\hline Mid-CCRT & 87 & 6.54 & 4.41 to 10.35 & & 616 & 414 to 910 & \\
\hline End-CCRT & 86 & 7.90 & 5.82 to 15.04 & & 377 & 234 to 525 & \\
\hline Head and neck cancer $[n=40]$ & & & & $<0.001$ & & & $<0.001$ \\
\hline Pre-CCRT & 40 & 2.08 & 1.58 to 2.93 & & 2,042 & 1,723 to 2,835 & \\
\hline Mid-CCRT & 40 & 7.76 & 6.00 to 10.56 & & 685 & 530 to 890 & \\
\hline End-CCRT & 40 & 7.58 & 4.63 to 12.87 & & 421 & 281 to 561 & \\
\hline Esophageal cancer $[n=28]$ & & & & $<0.001$ & & & $<0.001$ \\
\hline Pre-CCRT & 28 & 2.19 & 1.73 to 3.84 & & 2,101 & 1,786 to 2,542 & \\
\hline Mid-CCRT & 27 & 5.36 & 3.32 to 8.10 & & 526 & 229 to 926 & \\
\hline End-CCRT & 26 & 12.19 & 6.00 to 22.75 & & 255 & 140 to 538 & \\
\hline Cervical cancer $[n=20]$ & & & & $<0.001$ & & & $<0.001$ \\
\hline Pre-CCRT & 20 & 2.75 & 1.89 to 4.25 & & 2,123 & 1,261 to 2,634 & \\
\hline Mid-CCRT & 20 & 6.42 & 4.55 to 10.72 & & 560 & 360 to 1,064 & \\
\hline End-CCRT & 20 & 7.20 & 6.16 to 11.85 & & 377 & 315 to 489 & \\
\hline
\end{tabular}

Abbreviations: ALC, absolute lymphocyte count; CCRT, concurrent chemoradiotherapy; IQR, interquartile range; NLR, neutrophil to lymphocyte ratio; $P, P$-value from Friedman test to compare NLR and ALC between pre-CCRT, mid-CCRT, and end-CCRT.

\subsection{Ethics approval and consent to participate}

All subjects provided the institutional approved informed consents. This study was approved by each institutional ethics committee (i.e., Chiang Mai University Hospital, Prince Songklanagarind University Hospital and Maharaj Nakhonratchasima Hospital).

\subsection{NLR and ALC measurements}

NLR was calculated at each treatment visit (preCCRT, mid-CCRT, and end-CCRT) by dividing the percentage of neutrophils by the percentage of lymphocytes.

ALC was calculated as white blood cell count $\times$ $1,000 \times$ the percentage of lymphocytes at pre-CCRT, mid-CCRT, and end-CCRT.

NLR and ALC percent changes from pre-CCRT to mid-CCRT or end-CCRT: NLR or ALC \%change = (mid-CCRT or end-CCT value - pre-CCRT value/preCCRT value $) \times 100$.

\subsection{Statistical analysis}

Patient characteristics at pre-CCRT were compared between the two groups (group A and group B) using the Chi-square or Fisher's exact test for categorical variables and Mann-Whitney test for continuous variables. Median NLR and ALC were compared between the two groups (group A and group B) using the Mann-Whitney test. NLR and ALC between the three treatment vis- its (i.e., pre-CCRT, mid-CCRT, and end-CCRT) were compared using the Friedman test. Percent changes of NLR and ALC from pre-CCRT to mid-CCRT and endCCRT were compared between the two groups (group A and group B) using the Mann-Whitney test. Univariable and multivariable generalized estimation equation (GEE) models were used to assess the association between dynamic changes of NLR and ALC over time and the following characteristics: gender, cancer site (i.e., head and neck, esophageal, or cervical cancer), age, body mass index (BMI) at pre-CCRT, and study group (group A and group B). Clinically relevant variables or covariates from univariable analyses with $P<0.100$ were included in the multivariable models; covariates found to be collinear were excluded from the multivariable model. With the inclusion of the study groups and cancer sites being forced into the model, a backward model selection procedure was used to identify the multivariable model with the best overall fit.

\section{Results}

Patient characteristics at pre-CCRT, including age, gender, weight, height, body mass index, albumin, hemoglobin, tumor sites, tumor grade, tumor stage and Eastern Cooperative Oncology Group (ECOG) performance status were compared between groups A and B, as reported in Supplementary Table S1. There were no significant differences in characteristics at pre-CCRT between the two groups $(P>0.05)$. 


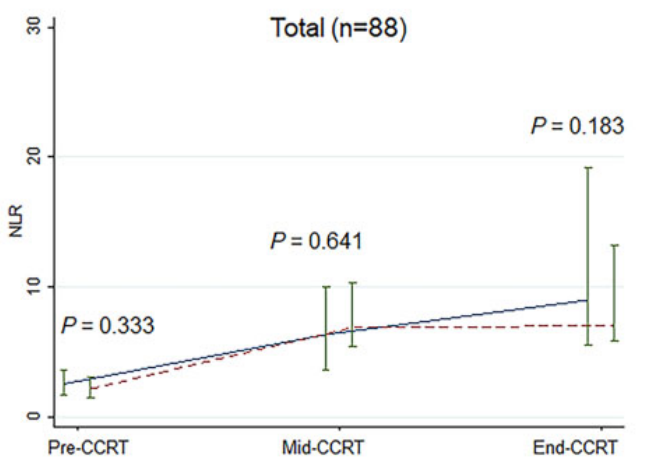

(a)

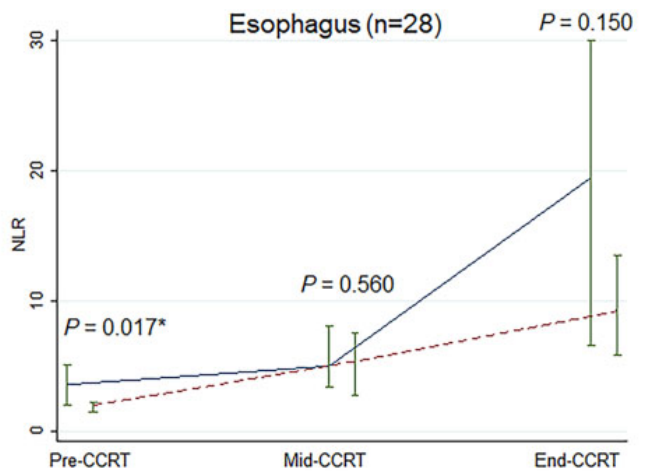

(c)

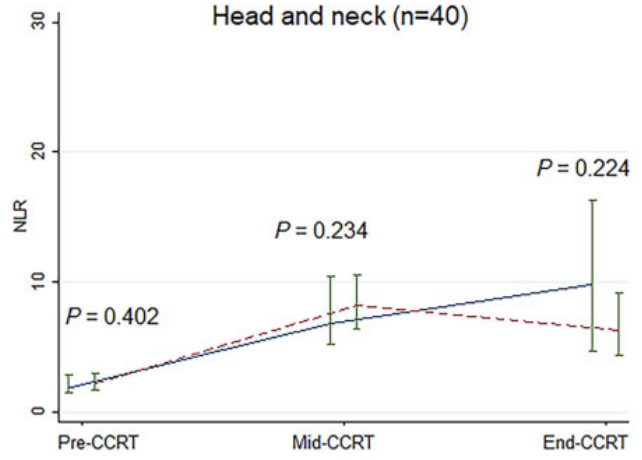

(b)

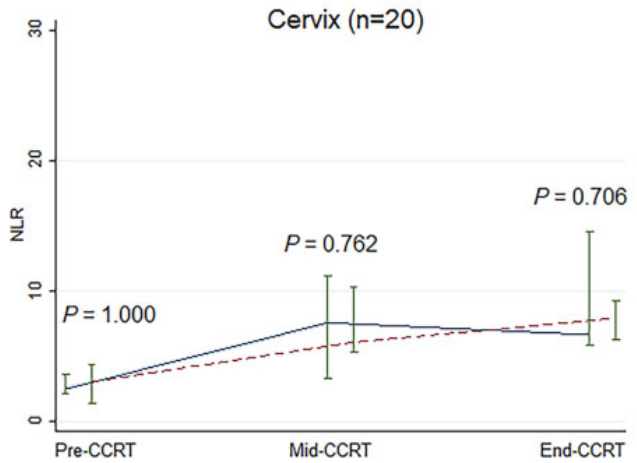

(d)

Fig. 1. Median and interquartile range of the neutrophil to lymphocyte ratio over time according to treatment visit for concurrent chemoradiotherapy. NLR, neutrophil to lymphocyte ratio; $P, P$-value from the Mann-Whitney test to compare NLR between two groups at each treatment visit; $* P<$ 0.05 .

Table 1 reports the medians for NLR and ALC at preCCRT compared with mid-CCRT and end-CCRT for all patients and each cancer site. Overall, median NLRs at mid-CCRT and end-CCRT were higher than those at pre-CCRT (6.54 at mid-CCRT and 7.90 at end-CCRT compared to 2.26 at pre-CCRT; $P<0.001)$. Meanwhile, ALCs at mid-CCRT and end-CCRT were significantly lower than the pre-CCRT level for all patients and each cancer site $(P<0.001)$.

As shown in Fig. 1, NLRs at pre-CCRT, mid-CCRT, and end-CCRT were not significantly different between groups A and B for all cancer sites, except that the median NLR value of esophageal cancer patients at preCCRT in group A was higher than in group B. However, the difference between the two groups was not statistically significant throughout the CCRT course (Fig. 1c). There were no statistically significant differences in the median ALCs between the two groups for overall patients, esophageal cancer patients, and cervical cancer patients (Fig. 2). For the head and neck cancer patients,
ALC at end-CCRT was higher in group B compared with group A $(P=0.023)$, as shown in Fig. $2 b$.

In Table 2, it can be seen that NLR increased throughout CCRT compared to pre-CCRT in both groups. The NLR value of patients in group B increased less, although the change was not statistically significant. ALC decreased during CCRT compared to pre-CCRT in the two groups; the decrease in patients in group B was less, although the change was not statistically significant.

A GEE model was used to identify factors associated with NLR and ALC changes over time in Table 3. At the multivariate level, NLR at pre-CCRT was independently associated with an increase in NLR over time. NLR during CCRT increased by 1.08 (95\% confidence interval [95\% CI]: 0.64-1.52) for each unit increase in pre-CCRT NLR in which a higher NLR level at preCCRT was significantly associated with a higher NLR after CCRT $(P<0.001)$.

After adjusting for the study groups, NLR at preCCRT, and cancer sites, we found that NLR at midCCRT increased 5.33 times compared to pre-CCRT, 
Table 2

Percentage changes in the neutrophil to lymphocyte ratio and absolute lymphocyte count from pre-CCRT to mid-CCRT and end-CCRT $(n=88)$

\begin{tabular}{|c|c|c|c|c|c|}
\hline \multirow[t]{2}{*}{ Variables } & \multicolumn{2}{|c|}{ Group A } & \multicolumn{2}{|r|}{ Group B } & \multirow[t]{2}{*}{$P$} \\
\hline & Median & Interquartile range & Median & Interquartile range & \\
\hline \multicolumn{6}{|l|}{ NLR } \\
\hline Pre-CCRT to mid-CCRT & 59.03 & 39.20 to 74.65 & 66.68 & 38.11 to 81.55 & 0.443 \\
\hline Pre-CCRT to end-CCRT & 75.51 & 37.75 to 85.78 & 69.79 & 59.46 to 79.17 & 0.458 \\
\hline \multicolumn{6}{|l|}{ ALC } \\
\hline Pre-CCRT to midCCRT & -243.71 & -411.84 to -113.39 & -262.26 & -407.73 to -144.13 & 0.502 \\
\hline Pre-CCRT to end-CCRT & -503.65 & -934.08 to -317.40 & -413.10 & -620.61 to -198.90 & 0.190 \\
\hline
\end{tabular}

Abbreviations: ALC, absolute lymphocyte count; CCRT, concurrent chemoradiotherapy; NLR, neutrophiltolymphocyte ratio; $P$, $P$-value from the Mann-Whitney testto compare NLR and ALC between two groups.

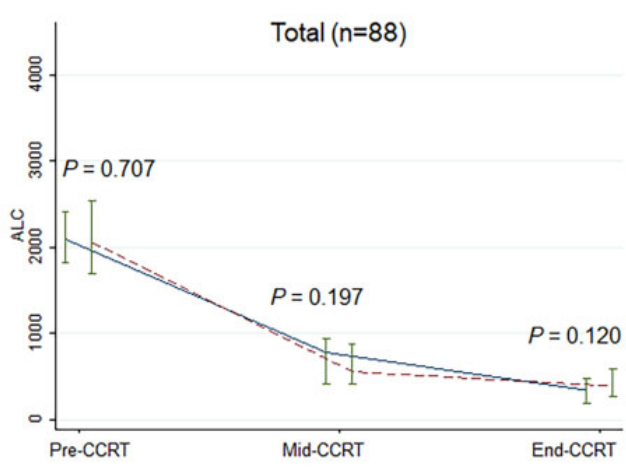

(a)

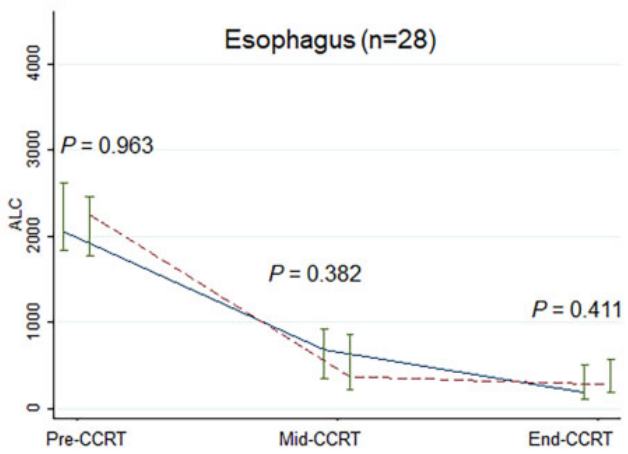

(c)

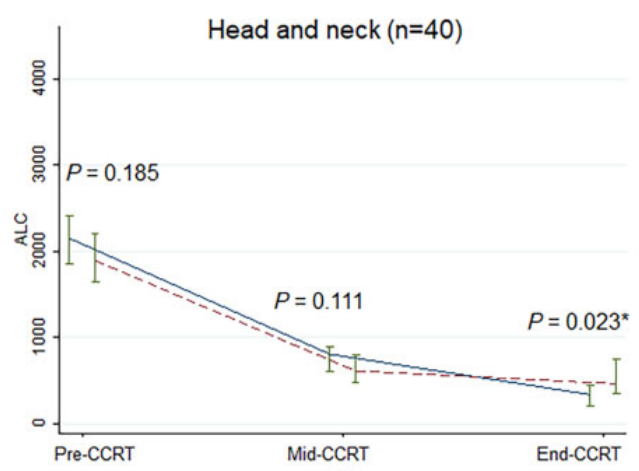

(b)

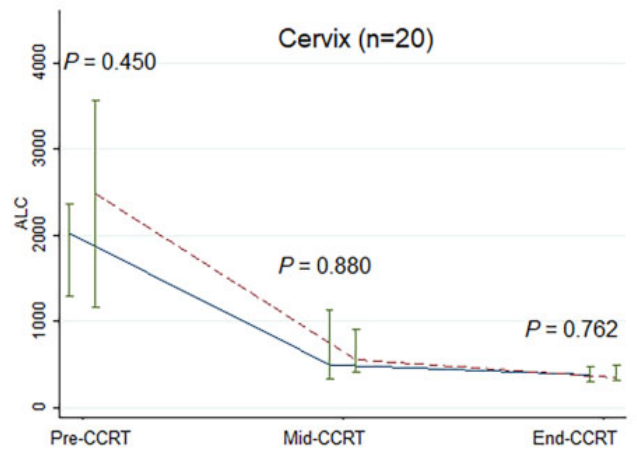

(d)

Fig. 2. Median and interquartile range of absolute lymphocyte count over time according to treatment visit of concurrent chemoradiotherapy. ALC, absolute lymphocyte count; $P, P$-value from the Mann-Whitney test to compare ALC between two groups at each treatment visit; $* P<0.05$.

and NLR at end-CCRT increased 8.79 times compared to pre-CCRT (adjusted $\beta=5.33$; 95\% CI: 3.34-7.32; $P<0.001$ and adjusted $\beta=8.79 ; 95 \%$ CI: 6.80 10.79; $P<0.001$, respectively). There was a lower rate of NLR change over time in group B than group A, although the difference was not statistically significant. Table 4 reports the univariable and multivariable models to identify factors associated with ALC changes over time. In the multivariable model, ALC after CCRT increased by 0.41 cells $/ \mu \mathrm{L}$ (95\% CI: 0.36 0.46 ) for each unit increase in pre-CCRT ALC in which a higher ALC at pre-CCRT was significantly associated with a higher ALC after CCRT $(P<0.001)$. ALC was significantly decreased by $1,463.87$ cells $/ \mu \mathrm{L}$ at midCCRT (adjusted $\beta=-1,463.87 ; 95 \% \mathrm{CI}:-1,598.49$ to $-1,329.25 ; P<0.001$ ), and $1,692.18$ cells $/ \mu \mathrm{L}$ at endCCRT (adjusted $\beta=-1,692.18 ; 95 \%$ CI: $-1,827.09$ to $-1,557.27 ; P<0.001)$ compared to pre-CCRT. 
Table 3

Factors associated with neutrophil to lymphocyte ratio change overtime using generalized estimation equation models

\begin{tabular}{|c|c|c|c|c|c|c|}
\hline \multirow[t]{2}{*}{ Variables } & \multicolumn{3}{|c|}{ Univariable analysis } & \multicolumn{3}{|c|}{ Multivariable analysis } \\
\hline & $\beta$ & $95 \%$ CI of $\beta$ & $P$ & $\operatorname{adj} \beta$ & $95 \% \mathrm{CI}$ of $\beta$ & $P$ \\
\hline \multicolumn{7}{|l|}{ Arm } \\
\hline \multicolumn{7}{|l|}{ Group A [ref.] } \\
\hline Group B & -1.57 & -3.48 to 0.34 & 0.107 & -1.29 & -2.96 to 0.39 & 0.132 \\
\hline NLR at pre-CCRT & 1.06 & 0.62 to 1.50 & $<0.001$ & 1.08 & 0.64 to 1.52 & $<0.001$ \\
\hline \multicolumn{7}{|l|}{ Treatment visits of CCRT } \\
\hline End-CCRT & 8.73 & 6.74 to 10.73 & $<0.001$ & 8.79 & 6.80 to 10.79 & $<0.001$ \\
\hline \multicolumn{7}{|l|}{ Cancer site } \\
\hline \multicolumn{7}{|l|}{ Cervical cancer [ref.] } \\
\hline Head and neck cancer & -0.56 & -3.01 to 1.89 & 0.653 & 0.76 & -1.44 to 2.96 & 0.498 \\
\hline Esophageal cancer & 0.83 & -1.81 to 3.47 & 0.537 & 1.21 & -0.36 to 4.31 & 0.097 \\
\hline \multicolumn{7}{|l|}{ Gender } \\
\hline \multicolumn{7}{|l|}{ Female [ref.] } \\
\hline Male & 0.12 & -1.82 to 2.07 & 0.901 & & & \\
\hline
\end{tabular}

Abbreviations: $95 \% \mathrm{CI}, 95 \%$ confidence interval; $\beta$, regression coefficient; adj $\beta$, adjusted regression coefficient; BMI, body mass index;

CCRT, concurrent chemoradiotherapy; NLR, neutrophil to lymphocyte ratio; $P, P$-value from the Wald test; ref., reference group.

Table 4

Factors associated with absolute lymphocyte count change overtime using generalized estimation equation models

\begin{tabular}{|c|c|c|c|c|c|c|}
\hline \multirow[t]{2}{*}{ Variables } & \multicolumn{3}{|c|}{ Univariable analysis } & \multicolumn{3}{|c|}{ Multivariable analysis } \\
\hline & $\beta$ & $95 \%$ CI of $\beta$ & $P$ & $\operatorname{adj} \beta$ & $95 \%$ CI of $\beta$ & $P$ \\
\hline \multicolumn{7}{|l|}{ Arm } \\
\hline \multicolumn{7}{|l|}{ Group A [ref.] } \\
\hline Group B & 3.20 & -144.08 to 150.48 & 0.966 & 4.78 & -66.87 to 76.42 & 0.896 \\
\hline ALC at pre-CCRT & 0.40 & 0.35 to 0.45 & $<0.001$ & 0.41 & 0.36 to 0.46 & $<0.001$ \\
\hline \multicolumn{7}{|l|}{$\begin{array}{l}\text { Treatment visits of CCRT } \\
\text { Pre-CCRT [ref.] }\end{array}$} \\
\hline Mid-CCRT & $-1,461.62$ & $-1,596.15$ to $-1,327.10$ & $<0.001$ & $-1,463.87$ & $-1,598.49$ to $-1,329.25$ & $<0.001$ \\
\hline End-CCRT & $-1,687.10$ & $-1,822.08$ to $-1,552.11$ & $<0.001$ & $-1,692.18$ & $-1,827.09$ to $-1,557.27$ & $<0.001$ \\
\hline \multicolumn{7}{|l|}{ Cancer site } \\
\hline Head and neck cancer & 12.64 & -174.52 to 199.81 & 0.895 & 24.65 & -66.51 to 115.82 & 0.596 \\
\hline Esophageal cancer & 30.46 & -172.41 to 233.34 & 0.769 & -61.35 & -160.51 to 37.80 & 0.225 \\
\hline Age [years] & -1.25 & -7.72 to 5.22 & 0.705 & & & \\
\hline $\mathrm{BMI}\left[\mathrm{kgm}^{2}\right]$ & 13.47 & -6.90 to 33.84 & 0.195 & & & \\
\hline \multicolumn{7}{|l|}{ Gender } \\
\hline Female [ref.] & & & & & & \\
\hline Male & -78.47 & -225.53 to 68.59 & 0.296 & & & \\
\hline
\end{tabular}

Abbreviations: $95 \%$ CI, 95\% confidence interval; $\beta$, regression coefficient; adj $\beta$, adjusted regression coefficient; ALC, absolute lymphocyte count; BMI, body mass index; CCRT, concurrent chemoradiotherapy; $P, P$-value from the Wald test; ref., reference group.

However, the degree of ALC change over time between group A and group B was not statistically significant. Nevertheless, in the head and neck cancer subgroup, ALC at end-CCRT in group B was significantly higher than in group A (median ALC of 465.95 cells $/ \mu \mathrm{L}$ vs. 342.80 cells $/ \mu \mathrm{L}(P=0.023)$, respectively). The results of GEE model for head and neck cancer patients showed the degree of NLR and ALC change over time between group A and group B were not statistically sig- nificant (Supplementary Tables S2 and S3). For the type of tumors (i.e., oropharynx, hypopharynx, nasopharynx, and oral), no statistical associations to NLR and ALC changes over time were detected.

\section{Discussion}

Several markers are routinely used to evaluate nutrition status of patients. Serum albumin is one of nutrition 
parameters commonly used in clinical practice which may predict prognosis of patients. For example, hepatocellular carcinoma patients with low serum albumin level resulted in a worsening liver function and poor clinical outcomes [36]. In addition, white blood cells including neutrophils and lymphocytes are frequently measured in cancer patients, particularly during active anticancer treatment. In this study, we demonstrated the dynamic changes of practical prognostic factors for inflammation and immunity by using NLR and ALC calculated from $\mathrm{CBC}$, which is a routine laboratory test. We also showed the effect of immune-enhancing nutritional supplementation on NLR and ALC during CCRT.

NLR significantly increased throughout the course of CCRT for all cancer types, which signifies increasing inflammation as an undesirable effect of both radiotherapy and chemotherapy treatment. Our finding is consistent with Sunpaweravong et al. [37], who evaluated the anti-inflammatory effect of immune-enhancing nutrition on esophageal cancer during CCRT by using C-reactive protein (CRP) and tumor necrosis factoralpha (TNF- $\alpha$ ) as markers; they found that both inflammatory markers rose during CCRT. Machon et al. [38] evaluated the efficacy of immune-enhancing nutritional supplementation at 5 days before CCRT and 5 days before each cycle of chemotherapy during radiotherapy of head and neck cancer. Their results show that the median CRP was lower after the first cycle of 5 days of supplementation compared to the baseline. However, they did not find a significant change in median CRP between the first cycle of immune-enhancing nutritional supplementation and the end of radiotherapy. Ou et al. [39] demonstrated similar result of significant rising of NLR through radiotherapy course and verified that post-RT NLR was associated with 5-year overall survival (OS) and progression-free survival (PFS) in nasopharyngeal cancer.

Median ALC at pre-CCRT of all patients in the present study was 2,070 cells $/ \mu \mathrm{L}$, which is within the normal range. However, ALC decreased significantly from its pre-CCRT level throughout the whole course of CCRT to 616 and 377 cells/ $\mu \mathrm{L}$ at mid-CCRT and endCCRT, respectively, in all patients. This occurrence was also seen for all three cancer sites. Our finding is consistent with a sub-cohort of head and neck cancer patients receiving CCRT in the study by Ho et al. [34]. This group of patients had statistically significant decreased white blood cell count, and neutrophil and lymphocyte percentages after CCRT; their mean ALC levels before and after CCRT were 1,400 cells $/ \mu \mathrm{L}$ and 300 cells $/ \mu \mathrm{L}$
$(P<0.001)$, respectively. Campian et al. [40] discovered that the total lymphocyte count dropped rapidly from the normal value before CCRT to $<500$ cells $/ \mu \mathrm{L}$ only 2 months after starting CCRT for head and neck cancer. This is in line with the ALC in the head and neck cancer patients in our study, which dramatically lowered during the course of CCRT from 2,042 cells $/ \mu \mathrm{L}$ at pre-CCRT to 685 and 421 cells/ $\mu \mathrm{L}$ at mid-CCRT and end-CCRT, respectively. Furthermore, we also had similar results for the sub-cohort of esophageal and cervical cancers.

Based on the results of our study, radiotherapy and chemotherapy had a significant adverse effect on NLR and ALC during CCRT. Radiotherapy induces both apoptosis and non-apoptotic cell death to cancer cells. Apoptosis is a power-driven form of cell death that is either controlled or initiated in reaction to particular stimuli (e.g., cytokines) [41] or stimulated by cellular stress or injury [42]. Turrel et al. [43] summarized that the decline in ALC observed in cancer patients could be caused by a decreased production of lymphocytes. Chemoradiotherapy also significantly increases inflammation, and damage-associated molecular patterns are present after tissue damage from the radiation and activated pro-inflammatory immune system [44]. Elevated inflammatory cytokines also have been reported in cancer treated with radiotherapy and chemotherapy $[45,46]$. In our multivariate analysis, pre-CCRT NLR and preCCRT ALC were associated with NLR and ALC values during CCRT, respectively. Moreover, the time point during CCRT was associated with both NLR and ALC levels. Thus, we should keep NLR at pre-CCRT as low as possible and ALC at pre-CCRT before CCRT as high as possible.

Apart from the inflammation marker, NLR is used as a prognostic factor in cancers. Higher pretreatment NLR is correlated with worse OS in oropharyngeal cancer treated and worse disease-free survival and OS in esophageal cancer with radiotherapy [47-49]. High post-RT NLR is associated with poor OS and PFS in nasopharyngeal cancer and higher recurrent rate in breast cancer [39,50]. Similarly, ALC is used as prognosis factor and nutritional status assessment. Low post-RT ALC links to the deteriorated OS in oropharyngeal cancer [51]. ALC is also used as a part of the formulae to evaluate nutritional status which also associates with prognosis in cancer patient. Serum albumin and ALC are used to calculate prognostic nutritional index (PNI). The same lab tests with an additional ratio of current weight and ideal body weight are used for Geriatric Nutritional Risk Index (GNRI). Caputo 
et al. [52] demonstrated that low PNI $(<31.3)$ was associated with poor OS in hepatocellular carcinoma treated with Sorafenib. Sasaki et al. [53] evaluated elderly patients with colorectal cancer underwent surgery and found the correlation between low GNRI $(\leqslant 98)$ and higher postoperative complications and lower OS. The benefit of immune-enhancing nutrition on NLR and ALC was not proven in this study. However, we found that the increase in NLR during CCRT was less steep in the immune-enhancing nutritional supplementation group. The drop in percentage change of ALC was also lower in patients who received the supplementation but the difference was not statistically significant. Still, in the head and neck cancer patients, ALC at the end-CCRT was significantly higher in patients receiving immune-enhancing nutrition compared to the control group. Since NLR and ALC are sensitive markers for several conditions (e.g., infection, surgery, cancer, etc.), changes in both markers for monitoring the effect of immune-enhancing nutritional supplementation might have been obscured by co-determiners. In addition, we enrolled patients with several different sites of cancer that can have different degrees of inflammation, thereby making our results inconsistent [54].

Another study using different and more novel inflammatory markers (CRP and TNF- $\alpha$ ) than in our study revealed the benefits of immune-enhancing nutritional supplementation [37]. The authors found that the rise in CRP/TNF- $\alpha$ ratio from its pre-CCRT level was significantly lower in the immune-enhancing nutrition group [37]. Our previous study on clinical outcomes also showed that the immune-enhancing nutritional supplementation group was accompanied by a lower grade of hematologic toxicity [9]. These results imply the benefit of immune-enhancing nutritional supplementation even though there was no significant difference in NLR and ALC levels in our recent study.

Immune-enhancing nutrition may be one of key facet in cancer management. Other novel treatment might also be beneficial. Metronomic approach may be associated with improved nutritional status in patients with head and neck cancer [55] as well as patients with hepatocellular carcinoma [56].

This study has some limitations, the first being the diversity of cancer sites, as mentioned previously, since the values of NLR and ALC vary in the different primary cancers $[57,58]$. A focused study on each particular cancer site might reveal more detail on the dynamic changes of these values. The second potential limitation is that we did not measure other more novel inflammation markers such as CRP, interleukin-6 (IL-
6 ), and TNF- $\alpha$. However, this could also be viewed as a strength in that we monitored the inflammation and immunity status of the patients with only a simple blood test (CBC) taken once a week during CCRT which was a real-world clinical practice circumstance. Formulae that assess immunonutrition status such as PNI or GNRI which use simple blood test (CBC, serum albumin) can also be used in further study.

Our results indicate that the pre-CCRT NLR and ALC values were affected by CCRT, thereby confirming the results of Machon et al. [38] that immune-enhancing nutritional supplementation before CCRT can lower these inflammation markers. A future study to confirm that immune-enhanced nutritional supplementation improves these pre-CCRT inflammation and immunity marker is being planned as a prospective trial.

The recovery of NLR and ALC after CCRT also a potential subject for further investigation. Since we did not measure ALC and NLR during follow-up after CCRT and immune-enhancing nutritional supplementation was stopped immediately at the end of CCRT, the effect of the supplementation in this period cannot be evaluated. This is one of the limitations of our study and it would be interesting to investigate whether allowing continual immune-enhancing nutritional supplementation would enable recovery from treatment-related lymphopenia caused by CCRT since it takes some time for the normal cells to recover from the effects of both treatment modalities.

\section{Conclusions}

Dynamic detrimental changes in NLR and ALC levels were demonstrated throughout CCRT. Pre-CCRT values of both markers were significant predictors for a favorable change. There were noticeable promising changes in both NLR and ALC values in patients receiving immune-enhancing nutritional supplementation, although the differences were not statistically significant. The effect of immune-enhancing nutritional supplementation on pre-CCRT NLR and ALC should be evaluated in a future randomized study.

\section{Acknowledgments}

We acknowledge Northern Thai Research Group of Radiation Oncology (NTRG-RO), Faculty of Medicine and Chiang Mai University for supporting the current study. The nutritional supplementation and blenderized diet in this study was supported by Thai Otsuka Pharmaceutical Company, Bangkok, Thailand [grant number: CNN-ISS-2013]. 


\section{Author contributions}

Conception: Imjai Chitapanarux and Nontiya Homkham Interpretation or analysis of data: Imjai Chitapanarux and Nontiya Homkham

Preparation of the manuscript: Imjai Chitapanarux, Nontiya Homkham, Pooriwat Muangwong, Veeradej Pisprasert, Patrinee Traisathit, Rungarun Jiratrachu and Pattawee Chottaweesak

Revision for important intellectual content: Imjai Chitapanarux, Nontiya Homkham, Pooriwat Muangwong, Veeradej Pisprasert and Patrinee Traisathit Supervision: Imjai Chitapanarux

\section{Supplementary data}

The supplementary files are available to download from http://dx.doi.org/10.3233/CBM-210086.

\section{References}

[1] J.S. Hong, L.H. Wu, L. Su, H.R. Zhang, W.L. Lv, W.J. Zhang and J. Tian, Effect of chemoradiotherapy on nutrition status of patients with nasopharyngeal cancer, Nutrition and Cancer 68 (2016), 63-69.

[2] T. Li, J. Lv, G. Zhu, J. Li, S.C. Zhu, J. Wang, L. Xing, D. Yang, C. Xie, L. Shen, R. Zhao, H. Shi and J. Lang, Enteral nutrition to improve nutritional status, treatment tolerance, and outcomes in patients with esophageal cancer undergoing concurrent chemoradiotherapy (CCRT): Results of a prospective, randomized, controlled, multicenter trial (NCT 02399306), American Society of Clinical Oncology 35 (2017), 4033-4033.

[3] I. Prieto, S. Montemuiño, J. Luna, M.V. de Torres and E. Amaya, The role of immunonutritional support in cancer treatment: Current evidence, Clinical Nutrition 36 (2017), 14571464.

[4] R.K. Chandra and S. Kumari, Effects of nutrition on the immune system, Nutrition 10 (1994), 207-210.

[5] L.M. Donini, C. Savina, L.M. Ricciardi, C. Coletti, M. Paolini, L. Scavone, M.R. De Felice, A. Laviano, F.R. Fanelli and C. Cannella, Predicting the outcome of artificial nutrition by clinical and functional indices, Nutrition 25 (2009), 11-19.

[6] M. Braga, P.E. Wischmeyer, J. Drover and D.K. Heyland, Clinical evidence for pharmaconutrition in major elective surgery, Journal of Parenteral and Enteral Nutrition 37 (2013), 66S$72 \mathrm{~S}$.

[7] R.F. Grimble, Basics in clinical nutrition: Immunonutrition nutrients which influence immunity: Effect and mechanism of action, European E-Journal of Clinical Nutrition and Metabolism 4 (2009), e10-e13.

[8] I. Chitapanarux, V. Pisprasert, E. Tharavichitkul, S. Jakrabhandu, P. Klunklin, W. Onchan, B. Supawongwattana, P. Traisathit, S. Rattanachaiwong and W.M. Sattasiri, Randomized study of nutritional status and treatment toxicities of oral arginine, glutamine, and omega- 3 fatty acids during concurrent chemoradiotherapy for head and neck cancer patients, Functional Foods in Health and Disease 6 (2016), 121-132.
[9] I. Chitapanarux, P. Traisathit, T. Chitapanarux, R. Jiratrachu, P. Chottaweesak, S. Chakrabandhu, W. Rasio, V. Pisprasert and P. Sripan, Arginine, glutamine, and fish oil supplementation in cancer patients treated with concurrent chemoradiotherapy: A randomized control study, Current Problems in Cancer 44 (2020), 100482

[10] C. Chuntrasakul, S. Siltharm, S. Sarasombath, C. Sittapairochana, W. Leowattana, S. Chockvivatanavanit and A. Bunnak, Metabolic and immune effects of dietary arginine, glutamine and omega-3 fatty acids supplementation in immunocompromised patients, Journal of the Medical Association of Thailand 81 (1998), 334-343.

[11] C. Grassberger, S.G. Ellsworth, M.Q. Wilks, F.K. Keane and J.S. Loeffler, Assessing the interactions between radiotherapy and antitumour immunity, Nature Reviews Clinical Oncology 16 (2019), 729-745.

[12] E. Abelardo, G. Davies, Y. Kamhieh and V. Prabhu, Are inflammatory markers significant prognostic factors for head and neck cancer patients? Journal for Oto-Rhino-Laryngology and Its Related Specialties 82 (2020), 235-244.

[13] Y. Cho, J.W. Kim, H.I. Yoon, C.G. Lee, K.C. Keum and I.J. Lee, The prognostic significance of neutrophil-to-lymphocyte ratio in head and neck cancer patients treated with radiotherapy, Journal of Clinical Medicine 7 (2018), 512.

[14] Q.T. Huang, Q.Q. Man, J. Hu, Y.L. Yang, Y.M. Zhang, W. Wang, M. Zhong and Y.H. Yu, Prognostic significance of neutrophil-to-lymphocyte ratio in cervical cancer: A systematic review and meta-analysis of observational studies, Oncotarget 8 (2017), 16755-16764.

[15] J. Wu, M. Chen, C. Liang and W. Su, Prognostic value of the pretreatment neutrophil-to-lymphocyte ratio in cervical cancer: A meta-analysis and systematic review, Oncotarget 8 (2017), $13400-13412$

[16] X. Xie, K.J. Luo, Y. Hu, J.Y. Wang and J. Chen, Prognostic value of preoperative platelet-lymphocyte and neutrophillymphocyte ratio in patients undergoing surgery for esophageal squamous cell cancer, Diseases of the Esophagus 29 (2016), 79-85.

[17] A.J. Templeton, M.G. McNamara, B. Seruga, F.E. VeraBadillo, P. Aneja, A. Ocana, R. Leibowitz-Amit, G. Sonpavde, J.J. Knox, B. Tran, I.F. Tannock and E. Amir, Prognostic role of neutrophil-to-lymphocyte ratio in solid tumors: A systematic review and meta-analysis, Journal of the National Cancer Institute 106 (2014), dju124.

[18] T. Kaya, S.B. Acikgoz, M. Yildirim, A. Nalbant, A.E. Altas and H. Cinemre, Association between neutrophil-to-lymphocyte ratio and nutritional status in geriatric patients, Journal of Clinical Laboratory Analysis 33 (2019), e22636.

[19] P. Lissoni, F. Brivio, L. Fumagalli, G. Messina, V. Ghezzi, L. Frontini, L. Giani, M. Vaghi, A. Ardizzoia and G.S. Gardani, Efficacy of cancer chemotherapy in relation to the pretreatment number of lymphocytes in patients with metastatic solid tumors, The International Journal of Biological Markers 19 (2004), 135-140.

[20] P.S.N. van Rossum, W. Deng, D.M. Routman, A.Y. Liu, C. $\mathrm{Xu}$, Y. Shiraishi, M. Peters, K.W. Merrell, C.L. Hallemeier, R. Mohan and S.H. Lin, Prediction of severe lymphopenia during chemoradiation therapy for esophageal cancer: Development and validation of a pretreatment nomogram, Practical Radiation Oncology 10 (2020), e16-e26.

[21] X. Zhou, X. Sun, W. Zhao, X. Fang and X. Wang, Prognostic significance of peripheral blood absolute lymphocyte count and derived neutrophil to lymphocyte ratio in patients with 
newly diagnosed extranodal natural killer/T-cell lymphoma, Cancer Management and Research 11 (2019), 4243-4254.

[22] C.A. Jacobson and A.S. LaCasce, Lymphoma: Risk and response after solid organ transplant, Oncology 24 (2010), 936944.

[23] J.Y. Blay, F. Chauvin, A. Le Cesne, B. Anglaret, D. Bouhour, C. Lasset, G. Freyer, T. Philip and P. Biron, Early lymphopenia after cytotoxic chemotherapy as a risk factor for febrile neutropenia, Journal of Clinical Oncology 14 (1996), 636-643.

[24] C. Borg, I. Ray-Coquard, I. Philip, G. Clapisson, N. BendrissVermare, C. Menetrier-Caux, C. Sebban, P. Biron and J.Y. Blay, CD4 lymphopenia as a risk factor for febrile neutropenia and early death after cytotoxic chemotherapy in adult patients with cancer, Cancer 101 (2004), 2675-2680.

[25] C.W. Choi, H.J. Sung, K.H. Park, S.Y. Yoon, S.J. Kim, S.C. Oh, J.H. Seo, B.S. Kim, S.W. Shin, Y.H. Kim and J.S. Kim, Early lymphopenia as a risk factor for chemotherapy-induced febrile neutropenia, American Journal of Hematology 73 (2003), 263266.

[26] J. Peron, C. Cropet, O. Tredan, T. Bachelot, I. Ray-Coquard, G. Clapisson, S. Chabaud, I. Philip, C. Borg, P. Cassier, I. Labidi Galy, C. Sebban, D. Perol, P. Biron, C. Caux, C. MenetrierCaux and J.Y. Blay, CD4 lymphopenia to identify end-of-life metastatic cancer patients, European Journal of Cancer 49 (2013), 1080-1089

[27] I. Ray-Coquard, C. Borg, T. Bachelot, C. Sebban, I. Philip, G. Clapisson, A. Le Cesne, P. Biron, F. Chauvin, J.Y. Blay and E.S. Group, Baseline and early lymphopenia predict for the risk of febrile neutropenia after chemotherapy, British Journal of Cancer 88 (2003), 181-186.

[28] I. Ray-Coquard, H. Ghesquière, T. Bachelot, C. Borg, P. Biron, C. Sebban, A. LeCesne, F. Chauvin and J.Y. Blay, Identification of patients at risk for early death after conventional chemotherapy in solid tumours and lymphomas, British Journal of Cancer 85 (2001), 816-822.

[29] R. Gamberale, C.M. Galmarini, P. Fernandez-Calotti, L. Jordheim, J. Sanchez-Avalos, C. Dumontet, J. Geffner and M. Giordano, In vitro susceptibility of CD4+ and CD8 + T cell subsets to fludarabine, Biochemical Pharmacology 66 (2003), 2185-2191.

[30] C. Tang, Z. Liao, D. Gomez, L. Levy, Y. Zhuang, R.A. Gebremichael, D.S. Hong, R. Komaki and J.W. Welsh, Lymphopenia association with gross tumor volume and lung V5 and its effects on non-small cell lung cancer patient outcomes, International Journal of Radiation Oncology Biology Physics 89 (2014), 1084-1091.

[31] P.W. Wijermans, W.B. Gerrits and H.L. Haak, Severe immunodeficiency in patients treated with fludarabine monophosphate, European Journal of Haematology 50 (1993), 292-296.

[32] J. Bauml, T.Y. Seiwert, D.G. Pfister, F. Worden, S.V. Liu, J. Gilbert, N.F. Saba, J. Weiss, L. Wirth, A. Sukari, H. Kang, M.K. Gibson, E. Massarelli, S. Powell, A. Meister, X. Shu, J.D. Cheng and R. Haddad, Pembrolizumab for platinum- and cetuximab-refractory head and neck cancer: Results from a single-arm, phase II study, Journal of Clinical Oncology $\mathbf{3 5}$ (2017), 1542-1549

[33] R.L. Ferris, G. Blumenschein, Jr., J. Fayette, J. Guigay, A.D. Colevas, L. Licitra, K. Harrington, S. Kasper, E.E. Vokes, C. Even, F. Worden, N.F. Saba, L.C. Iglesias Docampo, R Haddad, T. Rordorf, N. Kiyota, M. Tahara, M. Monga, M. Lynch, W.J. Geese, J. Kopit, J.W. Shaw and M.L. Gillison, Nivolumab for recurrent squamous-cell carcinoma of the head and neck, The New England Journal of Medicine 375 (2016), 1856-1867.
[34] W.J. Ho, M. Yarchoan, A. Hopkins, R. Mehra, S. Grossman and H. Kang, Association between pretreatment lymphocyte count and response to PD1 inhibitors in head and neck squamous cell carcinomas, Journal for Immunotherapy of Cancer 6 (2018), 84-91.

[35] T.Y. Seiwert, B. Burtness, R. Mehra, J. Weiss, R. Berger, J.P. Eder, K. Heath, T. McClanahan, J. Lunceford, C. Gause, J.D. Cheng and L.Q. Chow, Safety and clinical activity of pembrolizumab for treatment of recurrent or metastatic squamous cell carcinoma of the head and neck (KEYNOTE-012): An open-label, multicentre, phase 1b trial, Lancet Oncology 17 (2016), 956-965.

[36] A. Granito and L. Bolondi, Non-transplant therapies for patients with hepatocellular carcinoma and Child-Pugh-Turcotte class B cirrhosis, Lancet Oncology 18 (2017), e101-e112.

[37] S. Sunpaweravong, P. Puttawibul, S. Ruangsin, S. Laohawiriyakamol, P. Sunpaweravong, D. Sangthawan, J. Pradutkanchana, P. Raungkhajorn and A. Geater, Randomized study of antiinflammatory and immune-modulatory effects of enteral immunonutrition during concurrent chemoradiotherapy for esophageal cancer, Nutrition and Cancer 66 (2014), 1-5.

[38] C. Machon, S. Thezenas, A.M. Dupuy, E. Assenat, F. Michel, E. Mas, P. Senesse and J.P. Cristol, Immunonutrition before and during radiochemotherapy: Improvement of inflammatory parameters in head and neck cancer patients, Supportive Care in Cancer 20 (2012), 3129-3135.

[39] D. Ou, X. Wang, M. Wu, F. Xue, Y. Li, C. Hu and X. He, Prognostic value of post-radiotherapy neutrophil-to-lymphocyte ratio in locally advanced nasopharyngeal carcinoma, Strahlentherapie und Onkologie 196 (2020), 252-261.

[40] J.L. Campian, G. Sarai, X. Ye, S. Marur and S.A. Grossman, Association between severe treatment-related lymphopenia and progression-free survival in patients with newly diagnosed squamous cell head and neck cancer, Head \& Neck 36 (2014), 1747-1753.

[41] C.A. Smith, T. Farrah and R.G. Goodwin, The TNF receptor superfamily of cellular and viral proteins: Activation, costimulation, and death, Cell 76 (1994), 959-962.

[42] L.E. Gerschenson and R.J. Rotello, Apoptosis: A different type of cell death, FASEB Journal 6 (1992), 2450-2455.

[43] F. Turrel, C. Guignant, F. Venet, A. Lepape and G. Monneret, Innovative therapeutic strategies for restoring lymphocyte functions in septic patients, Inflammation \& Allergy Drug Targets 7 (2008), 181-186.

[44] Y. Shi, J.E. Evans and K.L. Rock, Molecular identification of a danger signal that alerts the immune system to dying cells, Nature 425 (2003), 516-521.

[45] S. Siva, M. MacManus, T. Kron, N. Best, J. Smith, P. Lobachevsky, D. Ball and O. Martin, A pattern of early radiation-induced inflammatory cytokine expression is associated with lung toxicity in patients with non-small cell lung cancer, PLoS One 9 (2014), e109560.

[46] X.S. Wang, Q. Shi, L.A. Williams, L. Mao, C.S. Cleeland, R.R Komaki, G.M. Mobley and Z. Liao, Inflammatory cytokines are associated with the development of symptom burden in patients with NSCLC undergoing concurrent chemoradiation therapy, Brain Behavior and Immunity 24 (2010), 968-974.

[47] S.P. Ng, H. Bahig, A. Jethanandani, E.M. Sturgis, F.M. Johnson, B. Elgohari, G.B. Gunn, R. Ferrarotto, J. Phan, D.I. Rosenthal, S.J. Frank, C.D. Fuller and A.S. Garden, Prognostic significance of pre-treatment neutrophil-to-lymphocyte ratio (NLR) in patients with oropharyngeal cancer treated with radiotherapy, British Journal of Cancer 124 (2021), 628-633. 
[48] C. Panje, O. Riesterer, C. Glanzmann and G. Studer, Neutrophil-lymphocyte ratio complements volumetric staging as prognostic factor in patients treated with definitive radiotherapy for oropharyngeal cancer, BMC Cancer 17 (2017), 643.

[49] A. Powell, C. Eley, C. Chin, A.H. Coxon, A. Christian, W.G. Lewis and South East Wales Oesophagogastric Cancer Collaborative, Prognostic significance of serum inflammatory markers in esophageal cancer, Esophagus 18 (2021), 267-277.

[50] C.I. Yoon, D. Kim, S.G. Ahn, S.J. Bae, C. Cha, S. Park, S Park, S.I. Kim, H.S. Lee, J.Y. Park and J. Jeong, Radiotherapyinduced high neutrophil-to-lymphocyte ratio is a negative prognostic factor in patients with breast cancer, Cancers 12 (2020), 1896.

[51] A.J. Lin, M. Gang, Y.J. Rao, J. Campian, M. Daly, H. Gay, P. Oppelt, R.S. Jackson, J. Rich, R. Paniello, J. Zevallos, D. Hallahan, D. Adkins and W. Thorstad, Association of posttreatment lymphopenia and elevated neutrophil-to-lymphocyte ratio with poor clinical outcomes in patients with human papillomavirusnegative oropharyngeal cancers, JAMA Otolaryngology - Head \& Neck Surgery 145 (2019), 413-421.

[52] F. Caputo, V. Dadduzio, F. Tovoli, G. Bertolini, G. Cabibbo, K. Cerma, C. Vivaldi, L. Faloppi, M.D. Rizzato, F. Piscaglia, C. Celsa, L. Fornaro, G. Marisi, F. Conti, N. Silvestris, M. Silletta, S. Lonardi, A. Granito, C. Stornello, V. Massa, G. Astara, S Delcuratolo, S. Cascinu, M. Scartozzi and A. Casadei-Gardini, The role of PNI to predict survival in advanced hepatocellu- lar carcinoma treated with Sorafenib, PLoS One 15 (2020), e0232449.

[53] M. Sasaki, N. Miyoshi, S. Fujino, T. Ogino, H. Takahashi, M. Uemura, C. Matsuda, H. Yamamoto, T. Mizushima, M. Mori and Y. Doki, The geriatric nutritional risk index predicts postoperative complications and prognosis in elderly patients with colorectal cancer after curative surgery, Scientific Reports 10 (2020), 10744

[54] P. Forget, G. Echeverria, S. Giglioli, B. Bertrand, S. Nikis, J.P. Lechat and M. De Kock, Biomarkers in immunonutrition programme, is there still a need for new ones? A brief review, Ecancermedicalscience 9 (2015), 546.

[55] P.M. Parikh, S.S. Hingmire and C.D. Deshmukh, Selected current data on metronomic therapy (and its promise) from India, South Asian Journal of Cancer 5 (2016), 37-47.

[56] A. Granito, S. Marinelli, E. Terzi, F. Piscaglia, M. Renzulli, L. Venerandi, F. Benevento and L. Bolondi, Metronomic capecitabine as second-line treatment in hepatocellular carcinoma after sorafenib failure, Digestive and Liver Disease 47 (2015), 518-522.

[57] R. Howard, P.A. Kanetsky and K.M. Egan, Exploring the prognostic value of the neutrophil-to-lymphocyte ratio in cancer, Scientific Reports 9 (2019), 19673.

[58] L.F. Porrata and S.N. Markovic, Is absolute lymphocyte count just another prognostic factor in cancer? SRX Medicine $\mathbf{2 0 1 0}$ (2010), 812304 